\title{
El flujo de efectivo descontado como método de valuación de empresas mexicanas en el periodo 2001-2007
}

Verónica Patricia

Rodríguez Vázquez

Departamento de Finanzas y Contaduría, Universidad de las Américas, Puebla veronicap.rodriguez@udlap.mx

\section{Jaquelina Marcela}

Aca Varela

Universidad de las Américas, Puebla

marcela_aca@hotmail.com

\section{Resumen}

El objetivo de esta investigación es valuar una muestra de cincuenta empresas de la Bolsa Mexicana de Valores mediante el modelo de flujo efectivo descontado durante un periodo de siete años, de 2001 a 2007, para comparar los resultados obtenidos con el valor de mercado. Para ello, los flujos de efectivo se proyectaron con la tasa de crecimiento del último Producto Interno Bruto (PIB) sectorial conocido y con la media geométrica del PIB sectorial, con la finalidad de conocer cuál de las dos opciones proporciona una mejor estimación de la tasa de crecimiento para las empresas. Entre los principales resultados se encontró que a pesar de que existe relación estadísticamente significativa entre los valores calculados con el modelo flujo de efectivo descontado (FED) y los valores de mercado de las empresas en la mayoría de los años y sectores analizados, el coeficiente $\beta_{1}$ no fue consistente. Por otra parte, se llegó a la conclusión de que la media geométrica del PIB sectorial proporcionó una mejor estimación de la tasa de crecimiento de las empresas y, como consecuencia, mayor asociación lineal entre los valores de mercado y los valores calculados con el modelo FED. 
Palabras clave: flujo de efectivo descontado, valuación de empresas, valor de mercado, Producto Interno Bruto (PIB).

\title{
Discounted cash flow as a valuation method of Mexican enterprises during the 2001-2007 period
}

\begin{abstract}
The objective of this investigation was to evaluate a 50-enterprise sample of the Mexican Stock Exchange by the discounted-cash-flow model for a 7 year period, from 2001 to 2007 , in order to compare the results obtained with the market value. To that end, the cash flows were projected with the most recent growth rate of the last known cross-sectioned GDP and the cross-sectioned GDP's geometric medium, with the objective of knowing which of the two options gives a better estimate of the enterprise's growth rate. We found that, despite the existence of a statistically significant relationship between the calculated values with the FED model and the market values of the enterprises in most of the years and sectors analyzed, the $\beta$ coefficient was not consistent. On the other hand, we concluded that the cross-sectioned GDP's geometric medium gave a better estimate of the enterprise's growth rate and as a consequence, a greater lineal association between the market values and the values calculated with the FED model.
\end{abstract}

Keywords: discounted cash flow, enterprise valuation, market value, Gross Domestic Product (GDP).

\section{Introducción}

La valuación de empresas juega un papel fundamental al momento de hacer alianzas, buscar aportes de capital, planear fusiones, adquisiciones, ventas y escisiones. Por ello, la importancia de valuar a las empresas surge por la necesidad de información que requieren los administradores, inversionistas y accionistas para la toma de decisiones. En la actualidad, existen diversas investigaciones de origen estadounidense y europeo sobre este tema que son sustentadas por destacados autores, entre los que se puede citar a Copeland, Koller y Murring (1994), Rapapport (1986), Fernández (2004) y Luehrman (1997); sin embargo, en México los trabajos relativos a la valuación de empresas son escasos.

Particularmente para el caso de México, Saavedra (2007: 104) señala que "El modelo de flujos de efectivo disponible considera que el valor de la empresa en mar- 
cha estará dado por el flujo de efectivo libre disponible que se espera genere en el futuro, descontado a una tasa que refleje los riesgos del negocio"; de igual forma, dentro de sus conclusiones comenta que el considerar variables como el flujo de efectivo, el costo de capital, la tasa de crecimiento, el valor de la empresas más allá del periodo explicito de pronóstico, entre las principales, hace que éste modelo sea más completo y más congruente en el cálculo del valor de la empresa.

En el estudio que realizó Saavedra (2007) aplicó el modelo FED por un periodo de diez años a 71 empresas que durante este lapso cotizaban en la Bolsa Mexicana de Valores, con la finalidad de obtener su valor y compararlo con el precio de mercado. Encontró que gran parte de los sectores analizados dieron como resultado alta correlación entre los valores arrojados por el modelo FED y el precio de mercado.

Para continuar con esa línea de estudio, en este trabajo se pretende encontrar evidencia estadística que demuestre el grado de relación existente entre los valores de las empresas arrojados por el modelo de flujo de efectivo descontado ${ }^{1} \mathrm{y}$ los valores de mercado de empresas que cotizan en la Bolsa Mexicana de Valores.

En esta investigación el modelo de flujo de efectivo disponible se aplicará para valuar a cincuenta empresas que hasta finales del 2007 cotizaban en la Bolsa Mexicana de Valores. Es necesario indicar que dichas empresas han logrado sobrevivir en la actualidad de la muestra que tomó Saavedra en su análisis. El periodo de estudio comprenderá del año 2001 a 2007; se analizarán los sectores: Comercio, Comunicaciones y transportes, Construcción, Industria extractiva, Industria de la transformación o manufactura, Empresas diversificadas o controladoras y Otros servicios. Cabe señalar que los flujos de efectivo se proyectarán con la tasa de crecimiento del último PIB sectorial conocido y con la media geométrica del PIB sectorial con la intención de conocer cuál de las dos opciones proporciona una mejor estimación de la tasa de crecimiento para las empresas

${ }^{1} \mathrm{El}$ modelo de flujo de efectivo descontado se conoce como flujo de efectivo disponible o flujo de efectivo libre. 


\section{Modelo de flujo de efectivo descontado}

En los últimos años el tema de valuación de empresas ha tomado gran importancia debido a que juega un papel fundamental para tomar decisiones acertadas en relación con fusiones, alianzas estratégicas, adquisiciones, aportes de capital, etc. De acuerdo con Saavedra (2007: 104): "El modelo de flujo de efectivo disponible considera que el valor de la empresa en marcha estará dado por el flujo de efectivo libre disponible que se espera genere en el futuro, descontado a una tasa que refleje los riesgos del negocio."

Así también, Nelson (2000) especifica que el análisis de flujo de efectivo descontado gira en torno de dos variables: los flujos de efectivo esperados en el futuro y la tasa de descuento. Además, señala que la tasa de descuento refleja el riesgo inherente de invertir en un negocio. Por lo tanto, los inversionistas requieren altos rendimientos cuando los riesgos son grandes. A su vez, López (2002) considera que "una característica de la técnica del descuento de flujos es que combina información financiera con información del mercado de capitales". La primera proviene de los estados contables de la firma como los balances, el estado de resultados y el estado de cambios en el capital contable; la segunda se refiere a la volatilidad de las acciones de la firma, el rendimiento que se espera del mercado accionario en su conjunto, el rendimiento libre de riesgo, entre otros.

$\mathrm{Al}$ respecto, Fernández (2008a) indica que los métodos basados en el descuento de flujos de fondos determinan el valor de la empresa a través de la estimación de los flujos de efectivo que generará en el futuro para luego descontarlos a una tasa apropiada según el riesgo de dichos flujos. Además, señala que en la actualidad, en general, se recurre a la utilización del modelo del flujo de efectivo descontado porque constituye el único método de valuación conceptualmente "correcto" —cada vez más utilizado-, que considera a la empresa como un ente generador de flujos de fondos y, por ello, sus acciones y su deuda son valuados como otros activos financieros. De la misma forma, menciona que los métodos de descuento de flujos se basan en un pronóstico detallado y cuidadoso para cada periodo de cada una de las partidas financieras vinculadas a la generación de los flujos de efectivo correspondientes a las operaciones de la empresa. En este mismo sentido Vélez-Pareja y Tham (2003) afirman que el valor de la empresa (D+E) es igual al valor presente de los flujos de efectivo libres descontados al costo de capital promedio ponderado. 
Por su parte, López (2000) señala que el flujo de efectivo es la materia prima principal para calcular el valor de una firma, medir la rentabilidad de un proyecto de inversión, planificar las operaciones o establecer la capacidad de pago de una deuda, a lo que las entidades financieras suelen prestar mucha atención.

Copeland (2000: 167) menciona que "el flujo de efectivo libre es el verdadero flujo de efectivo operativo de una compañía, porque es el flujo de efectivo después de impuestos que está disponible para todos los accionistas y acreedores". Vélez-Pareja (2007) a su vez determina que el flujo de efectivo libre es lo que está disponible para los dueños de capital (acreedores o accionistas). En contraste, Fernández (2008b) comenta que el flujo de efectivo libre no es el flujo disponible para la deuda y las acciones. Por definición, el flujo disponible para la deuda y las acciones es el flujo de efectivo de capital $^{2}$. El flujo de efectivo libre es el hipotético flujo disponible para las acciones en el caso de que la empresa no tuviera deuda. Por lo tanto, Fernández (2008b) especifica que el flujo de efectivo libre es el flujo de fondos operativo, esto es el flujo de fondos generado por las operaciones, sin tener en cuenta el endeudamiento (deuda financiera), después de impuestos. Asimismo, López (2000) menciona que una de las técnicas comúnmente utilizadas para la valuación de empresas es el descuento del flujo de efectivo esperado por el costo promedio ponderado del capital (WACC) que corresponde al riesgo de ese flujo. Además, señala que el flujo de efectivo libre es el flujo del proyecto o negocio prescindiendo del financiamiento; en este sentido, representa el flujo de efectivo de la firma como si ésta se financiara enteramente con capital propio.

\section{Aplicación del modelo del flujo de efectivo descontado}

Diversos investigadores han modificado el modelo de flujo de efectivo descontado con la finalidad de aplicarlo en valuaciones específicas. De acuerdo con Luehrman (1997), el modelo de flujo de efectivo descontado se ha vuelto obsoleto, por ello este autor sugiere valuar a las empresas mediante el método del valor presente ajustado (APV) ${ }^{3}$, el cual valúa a las empresas, en primer lugar, como si estuvieran financiadas sólo con capital (flujos de efectivo descontados al costo de capital propio) y luego agrega los efectos del apalancamiento para reflejar la protección

\footnotetext{
${ }^{2}$ Se denomina flujo de efectivo de capital (capital cash flow) a la suma del flujo de efectivo para los poseedores de deuda más el flujo de efectivo para las acciones. El flujo de efectivo para los poseedores de deuda se compone de la suma de los intereses más devolución del principal. Fernández (2008:4)

${ }^{3}$ Adjusted Present Value, por sus siglas en ingles (APV)
} 
fiscal de la deuda (intereses deducibles descontados al costo de la deuda). Al respecto, Sabal (2007) indica que el APV proporciona buenas aproximaciones del valor cuando se utiliza en compañías pequeñas con razones de deuda inestables en países con legislación fiscal compleja y en mercados emergentes donde existe alta incertidumbre económica.

Berkman (2000) elaboró una investigación donde comparó el valor estimado derivado de los flujos de efectivo descontados (DFC) y el obtenido por el método de valuación precio-utilidad $(\mathrm{P} / \mathrm{E})$ con el precio de mercado para obtener un error de valuación. En dicho estudio se valuaron 45 empresas de la Bolsa de Valores de Nueva Zelanda entre 1989 y 1995 . En este estudio se encontró que el método de flujos de efectivos descontados y el precio-utilidad tienen una precisión similar; la media absoluta del error en el precio fue aproximadamente del $20 \%$ y los modelos explicaron alrededor del $70 \%$ de la variación del promedio representativo en el precio de mercado escalado por el valor en libros. La evidencia presentada en esta investigación apoya el uso de técnicas tradicionales de valuación de capital. Los resultados indican que los modelos DFC y P/E explican tres cuartos de la variación del promedio representativo en los precios de mercado.

Por otra parte, Desmet (2000) señala que las empresas de Internet están creciendo rápidamente; como consecuencia propone usar el modelo de flujo de efectivo descontado reforzado con un análisis microeconómico y escenarios de probabilidades para valuar a empresas con acciones de alta volatilidad, como son las compañías de Internet. En contraste, Athanassakos (2007) menciona que el método de valuación de flujo de efectivo descontado se aplica fácilmente en compañías estables; sin embargo, señala que este método no es útil para valuar inversiones con oportunidades de crecimiento substanciales (empresas de Internet); por lo tanto, los altos crecimientos en las empresas de Internet deben valuarse como opciones y, más específicamente, como una opción real.

De acuerdo con James y Koller (2000), los procedimientos para descontar los flujos de efectivo futuros al costo de capital promedio ponderado son iguales en cualquier lugar; pero en los mercados emergentes los riesgos son mayores; consecuencia de lo anterior, la valuación se vuelve más compleja en estos mercados porque los compradores y vendedores encaran mayores riesgos y obstáculos. Estos riesgos pueden incluir altos niveles de inflación, volatilidad macroeconómica, cambios políticos, guerras, cambios reglamentarios, pocos controles contables y corrup- 
ción. Por lo anterior, dichos autores sugieren que en los mercados emergentes se utilice el modelo del flujo de efectivo descontado en combinación con escenarios de probabilidades.

Por su parte, Fernández (2004) propone que las compañías temporales mediante el modelo de flujo de efectivo descontado se valúen utilizando datos mensuales; afirma que si se utilizan datos anuales será necesario hacer algunos ajustes; además, señala que la temporalidad se da principalmente por las ventas - como en el caso de fábricas de juguetes - o por las compras —en el caso de productores de aceite comestible. Cuando se valúan compañías, la temporalidad afecta el cálculo de los flujos de efectivo libres principalmente por el incremento de los requerimientos del capital de trabajo.

Heer y Koller (2000) sugieren que para la valuación de compañías cíclicas ${ }^{4}$-aerolíneas, químicas, papel y acero- se utilice el modelo de flujo de efectivo descontado basado en probabilidades para obtener estimaciones razonables.

En México el estudio más reciente sobre valuación de empresas mediante el modelo del flujo de efectivo disponible es el que realizó María Luisa Saavedra (2007); en su investigación aplicó el modelo FED a 71 empresas para el periodo de 1991 a 2000 que durante ese lapso cotizaban en la Bolsa Mexicana de Valores, con la finalidad de obtener su valor y compararlo con el precio de mercado. El flujo de efectivo se calculó como utilidad de operación menos impuestos, más depreciación y amortización. Se utilizó como tasa de crecimiento al PIB sectorial y como tasa de descuento al costo de capital promedio ponderado. El valor de negocio en marcha se cálculo dividiendo el último flujo de efectivo entre el CCPP (Costo de Capital Promedio Ponderado) menos la tasa de crecimiento. Una vez que se obtuvieron las variables necesarias para valuar a las empresas se proyectaron los flujos de efectivo por cinco años con el PIB sectorial, estos flujos se descontaron con la tasa de capital promedio ponderado y, finalmente, el valor de la empresa se calculó como la suma de los flujos de efectivo descontados al cCPP.

\footnotetext{
${ }^{4}$ Las empresas cíclicas mantienen un alto grado de variabilidad de sus ingresos.
} 
Se encontró que el modelo del flujo de efectivo disponible fue adecuado para valuar empresas, porque gran parte de los sectores analizados ${ }^{5}$ dieron como resultado una alta correlación entre los valores arrojados por el modelo FED y el precio de mercado, únicamente el sector de construcción fue el que mostró una correlación poco significativa. Finalmente, se llegó a la conclusión de que los valores arrojados por el modelo FED fueron consistentes con el precio de mercado.

De la misma forma, Adam (2005) realizó una investigación en la cual estudió el valor de las empresas y la creación de valor en el transcurso del tiempo. En dicho estudio se analizaron los estados financieros a fechas de cierre en series continuas de los años 1992-1997, 1997-2002 y 1992-2002 de las empresas de los sectores Alimentos, bebidas y tabaco; Comercio; Comunicaciones; Construcción; Controladores; Industria de la transformación y Otros servicios. Se valuaron 19 empresas de la Bolsa Mexicana de Valores mediante los siguientes métodos de valuación: el método de valor en libros, el método de valor de mercado, el método Goodwill, el modelo Black and Scholes, el método de flujos de efectivo descontado y el método del múltiplo precio-utilidad. Para los efectos de la investigación en Adam el problema de las proyecciones se subsanó por la misma naturaleza de la investigación debido a que se tomaron los flujos reales generados por las mismas empresas.

Con base en los resultados arrojados por cada modelo se concluyó que el método de valor de mercado es el que, en promedio, valoró más alto a las empresas, seguido del método precio-utilidad, ambos por arriba del valor en libros. Inferior a este valor estuvieron los arrojados por el método Goodwill, el modelo de Black and Scholes; en cambio el flujo descontado fue el método que menor valor le otorgó a las empresas.

\section{Metodología para el cálculo del modelo de flujo de efectivo descontado para determinar el valor de la empresa}

Las empresas se clasificaron de acuerdo con la división de la Bolsa Mexicana de Valoras en los sectores: Comercio, Comunicaciones y Transportes, Construcción, Industria extractiva, Transformación o Manufactura, Empresas Diversificadas y Otros servicios.

\footnotetext{
${ }^{5}$ Los sectores son: Alimentos, bebidas y tabaco con correlación de .86; Comercio con correlación de .81; Comunicaciones y transportes con correlación de .37; Empresas diversificadas o controladoras con correlación de .65; Industria de la transformación I con correlación de .81; Industria de la transformación II con correlación de .87; Servicios financieros con correlación de .41 y Otros servicios con correlación de .75 (Saavedra, 2008:103).
} 
El procedimiento que se llevó a cabo para valuar a las empresas mediante el modelo del flujo de efectivo descontado fue el siguiente:

1. Se proyectó el flujo de efectivo actual por cinco años mediante la tasa de crecimiento del PIB sectorial.

2. Se estimó el costo de capital promedio ponderado.

3. Se calculó el valor del negocio en marcha o perpetuidad.

4. Se descontó cada flujo de efectivo pronosticado y el valor del negocio en marcha al costo de capital promedio ponderado.

5. El valor de la empresa fue igual al valor presente de los flujos de efectivo proyectados más el valor presente de la perpetuidad (valor del negocio en marcha).

Cálculo de las variables del modelo del flujo de efectivo disponible

Las variables que se consideraron para valuar a las empresas que cotizan en la BMV mediante el FED son: flujo de efectivo, tasa de crecimiento, tasa de descuento y valor del negocio en marcha o perpetuidad.

Flujo de efectivo

El flujo de efectivo se calculó como:

$$
F . E=U . O-I+D-\Delta C . T
$$

Donde:

$F . E=$ Flujo de efectivo

$U . O=$ Utilidad de operación

$I=$ Impuestos

$D=$ Depreciación y amortización.

$\Delta C . T=$ Incremento en el capital de trabajo neto

El incremento en el capital de trabajo neto fue igual a la diferencia entre el activo circulante y el pasivo circulante del año actual menos la diferencia de éstos en el año anterior, es decir: 


$$
\Delta C . T .=\left(A . C_{t}-P . C_{t}\right)-\left(A . C_{t-1}-P . C_{t-1}\right)
$$

Los datos que se emplearon para calcular el flujo de efectivo se obtuvieron de la base de datos de Economática mediante el balance general y estado de resultados de cada empresa para cada año de análisis.

\section{Tasa de crecimiento}

De acuerdo con Saavedra (2007), las empresas no crecen más que la economía en general por periodos largos, por lo que sugiere que la mejor estimación de crecimiento sea el PIB ${ }^{6}$ en el largo plazo, teniendo un mejor acercamiento en el pronóstico si se utiliza el PIB sectorial. Lo anterior puede ser cuestionable, no obstante consideraremos el PIB como la mejor estimación de la esperanza del crecimiento de las empresas. Las tasas de crecimiento de las empresas representadas por el PIB sectorial se obtuvieron de los reportes anuales del Banco de México (Banxico).

Es importante mencionar que en primer lugar los flujos de efectivo se proyectaron con la tasas de crecimiento del último PIB sectorial conocido y posteriormente utilizando una media geométrica del PIB sectorial. Lo anterior, se efectuó con la finalidad de conocer cuál de las dos opciones proporcionaba una mejor estimación de la tasa de crecimiento para las empresas.

Para efectos de esta investigación se utilizó la siguiente fórmula para calcular la media geométrica del PIB sectorial:

$$
\bar{P} I \bar{B}_{G}=\left[\left(1+P I B_{1}\right) \times\left(1+P I B_{2}\right) \times\left(1+P I B_{3}\right) \times\left(1+P I B_{4}\right) \times\left(1+P I B_{5}\right)\right]^{\frac{1}{5}}-1
$$

Para obtener la media geométrica del PIB sectorial se tomó el PIB del año en el cual se quiere obtener el valor de la empresa y el PIB de cuatro años anteriores.

\footnotetext{
${ }^{6}$ El Producto Interno Bruto mide la renta de un país. Mankiw (2004: 312) define al PIB como "el valor de mercado de todos los bienes y servicios finales producidos en un país durante un determinado periodo".
} 


\section{Tasa de descuento}

La tasa de descuento que se empleó para traer a valor presente los flujos de efectivo fue el costo de capital promedio ponderado. El costo de capital promedio ponderado $^{7}$ se calculó como:

Donde:

$$
C C P P=\frac{V_{d}}{V_{d}+V_{e}} k_{d}(1-T)+\frac{V_{e}}{V_{d}+V_{e}} k_{e}
$$

$V_{d}=$ Valor de la deuda

$k_{d}=$ Costo de la deuda con costo

$V_{e}=$ Valor del capital

$k_{e}=$ Costo del capital

$T=$ Tasa de impuestos

La estimación del costo de la deuda $k_{d}$ se basó en las tasas de interés sobre la deuda de la empresa. Este costo se expresó después de impuestos, puesto que los pagos de intereses son fiscalmente deducibles.

El costo del capital propio $k_{e}$ se obtuvo mediante el Método del Modelo de Valuación de Activos de Capital, mejor conocido como $\mathrm{CAPM}^{8}$. En este trabajo se utilizó la tasa de $\operatorname{Cetes}^{9}$ a 364 días que proporciona el Banco de México como tasa libre de riesgo. Además, se tomó la beta apalancada de cada empresa que suministra Economática porque esta cifra refleja el riesgo adicional en el incurre una empresa cuando en su estructura de capital hay deuda. Finalmente, el rendimiento del mercado fue igual al cambio porcentual del IPC ${ }^{\text {Io }}$, el cual se obtendrá de los informes anuales de la Bolsa Mexicana de Valores.

\footnotetext{
${ }^{7}$ Suponiendo que las empresas no tienen acciones preferentes en su estructura de capital.

${ }^{8}$ CAPM por sus siglas en inglés (Capital Asset Pricing Model)

${ }^{9}$ Los Cetes son instrumentos de deuda emitidos por el gobierno federal.

${ }^{10}$ De acuerdo con la definición de la BMV el Índice de Precios y Cotizaciones es el principal indicador de la Bolsa Mexicana de Valores, expresa el rendimiento del mercado accionario en función de las variables de precios de una muestra balanceada ponderada y representativa del conjunto de acciones cotizadas en la Bolsa.
} 


\section{Valor de negocio en marcha o perpetuidad}

Se calculó como una perpetuidad, pues se asume que las empresas tienen un valor más allá del periodo explicito del pronóstico Siu (2001: 104).

En su aplicación más general se tiene:

$$
\text { Perpetuidad }=\frac{F E L_{T+1}}{(T D-g)}
$$

Donde:

$$
\begin{array}{ll}
F E L_{T+1}= & \text { Flujo de efectivo libre del año siguiente al } \\
& \text { periodo explicito de pronóstico } \\
T D & =\text { Tasa de descuento }(\mathrm{CCPP}) \\
g \quad & =\text { Tasa de crecimiento }
\end{array}
$$

\section{Cálculo del valor de la empresa}

El valor de la empresa se calculó como el valor presente de los flujos de efectivo libres más el valor presente de la perpetuidad. Para ser consistentes con la definición de flujos de efectivo libres, el valor presente de éstos durante y después del periodo explícito se obtuvo al descontar dichos flujos al costo de capital promedio ponderado.

$$
V E=\sum \frac{F E L}{(1+C C P P)^{n}} \quad+\text { Valor presente de la perpetuidad }
$$

Donde:

$V E=$ Valor de la empresa

$F E L=$ Flujo de efectivo libre de cada año de pronóstico

$C C P P=$ Costo de capital promedio ponderado

$n \quad=$ Periodos de pronóstico 


\section{Regresión lineal simple}

Las regresiones se efectuaron con la herramienta de análisis de datos de Microsoft Excel. Con la finalidad de encontrar evidencia suficiente que permita concluir con mayor certeza si los valores de las empresas se relacionan con los valores calculados con el modelo FED, se realizaron regresiones para cada empresa, año y sector en donde se llevó a cabo la valuación. Estas regresiones se aplicaron tanto para los valores de las empresas que se obtuvieron al utilizar el último PIB sectorial conocido como para aquellos que tomaron la media geométrica del PIB sectorial para proyectar los flujos de efectivo. Es importante indicar que los valores de mercado de las empresas se obtuvieron de Economática; para ello, se tomó como variable dependiente $(y)$ los valores de las empresas calculados con el FED debido a que éstos son los que se van a proyectar y como variable independiente $(x)$ los valores de mercado.

En consecuencia, debido a que se valuaron cincuenta empresas mediante el modelo FED con las dos diferentes estimaciones del PIB sectorial; es decir, el último conocido y la media geométrica, se efectuaron cien regresiones; además, como siete sectores se valuaron, se efectuaron un total de 14 regresiones. Asimismo, como se analizaron siete años, se realizaron 14 regresiones porque el periodo de estudio fue de 2001 a 2007. Por último, se realizaron dos regresiones globales.

\section{Resultados}

Después de aplicar la metodología planteada, se determinaron los valores de las empresas que conforman la muestra para cada uno de los siete años analizados. Los valores de mercado que se compararon con los valores que se obtuvieron con el modelo FED se pueden observar en el anexo 1. Por otra parte, los resultados de los cálculos de los valores de las empresas con el modelo FED al utilizar el último PIB sectorial conocido y los arrojados por la media geométrica del PIB sectorial, como tasa de crecimiento, se muestran en los anexos 2 y 3 .

\section{Resultados de las regresiones para cada empresa}

Como se planteó anteriormente, se realizaron cincuenta regresiones entre los valores calculados de las empresas con el modelo FED con el último PIB sectorial conocido y los valores de mercado. Además, se hicieron otras cincuenta regresiones 
para conocer la relación entre los valores calculados de las empresas con la media geométrica del PIB sectorial y los valores de mercado. Es importante destacar que en cada regresión se analizaron siete observaciones.

En el cuadro 1 se presenta un resumen de las regresiones donde existe una relación significativa entre los valores calculados de las empresas con el modelo FED y los valores de mercado.

\section{Cuadro 1}

Resumen de regresiones con relación estadísticamente significativa

\begin{tabular}{lc|lc}
\hline \multicolumn{2}{c}{$\begin{array}{c}\text { Valores calculados con el último PIB } \\
\text { sectorial conocido y valores de mercado }\end{array}$} & \multicolumn{2}{c}{$\begin{array}{c}\text { Valores calculados con la media geométrica } \\
\text { del PIB sectorial y valores de mercado }\end{array}$} \\
\hline $\begin{array}{c}\text { Empresas } \\
\text { con relación significativa }\end{array}$ & $\begin{array}{c}\text { Pendiente } \\
\text { de la recta }\left(\beta_{l}\right)\end{array}$ & $\begin{array}{c}\text { Empresas } \\
\text { con relación significativa }\end{array}$ & $\begin{array}{c}\text { Pendiente } \\
\text { de la recta }\left(\beta_{I}\right)\end{array}$ \\
\hline Organización Soriana & 10.53 & Grupo Casa Saba & 6.70 \\
Grupo Casa Saba & 3.47 & Corporativo Fragua & 4.99 \\
Corporativo Fragua & 2.50 & Cementos Chihuahua & 3.60 \\
Grupo Gigante & -3.09 & Empresa Ica & -1.23 \\
Teléfonos de México & -15.55 & Embotelladoras Unidas & 2.67 \\
Grupo Radio Centro & -13.40 & Tekchem & 12.32 \\
Grupo Iusacell & 29.77 & Cemex & 15.20 \\
Cementos Chihuahua & 1.92 & Grupo México & 2.67 \\
Empresa Ica & -9.77 & & \\
Médica Sur & 4.05 & & \\
\hline
\end{tabular}

Con base en los resultados de las regresiones anteriores se puede concluir que el modelo del flujo de efectivo disponible o descontado no proporcionó información convincente porque en pocas compañías se encontró relación significativa entre los valores calculados con este modelo y los valores de mercado. Sin embargo, Levine et. al (2006: 213) indican que el tamaño de la muestra debe ser de por lo menos treinta para que la distribución muestral se incline a seguir una distribución normal y se pueda utilizar la prueba $t$ para obtener conclusiones acertadas sobre las hipótesis. Por lo tanto, debido a que sólo se observaron los valores de las empresas durante un periodo de siete años, no se puede suponer que se sigue una distribución aproximadamente normal y mucho menos se puede garantizar que los resultados que se obtuvieron son válidos 
Resultados de las regresiones para cada año de análisis

En este bloque se hicieron un total de 14 regresiones; es decir, se elaboraron siete regresiones entre los valores calculados con el último PIB sectorial conocido y los valores de mercado y otras siete para conocer la relación entre los valores calculados con la media geométrica y los valores de mercado. Cabe mencionar que en cada regresión se analizaron cincuenta observaciones.

En cuadro 2 se pueden observar los años en los cuales se encontró una relación significativa entre los valores de mercado de las empresas y los valores calculados con el modelo FED.

\begin{tabular}{|c|c|c|c|}
\hline \multicolumn{4}{|c|}{$\begin{array}{l}\qquad \text { Cuadro } 2 \\
\text { Regresiones anuales con relación estadísticamente significativa }\end{array}$} \\
\hline \multicolumn{2}{|c|}{$\begin{array}{c}\text { Valores calculados con el último } \\
\text { PIB sectorial conocido y valores de } \\
\text { mercado de las empresas }\end{array}$} & \multicolumn{2}{|c|}{$\begin{array}{c}\text { Valores calculados con la media } \\
\text { geométrica del PIB sectorial y valores } \\
\text { de mercado de las empresas }\end{array}$} \\
\hline $\begin{array}{l}\text { Años con relación } \\
\text { significativa }\end{array}$ & $\begin{array}{c}\text { Pendiente } \\
\text { de la recta }\left(\beta_{1}\right)\end{array}$ & $\begin{array}{l}\text { Años con relación } \\
\text { significativa }\end{array}$ & $\begin{array}{c}\text { Pendiente de la } \\
\text { recta }\left(\beta_{1}\right)\end{array}$ \\
\hline 2001 & 1.95 & 2001 & 13.45 \\
\hline 2003 & 1.16 & 2002 & -6.58 \\
\hline 2004 & 0.56 & 2003 & 1.15 \\
\hline 2005 & 1.01 & 2004 & 0.44 \\
\hline 2006 & 0.85 & 2005 & 0.90 \\
\hline \multirow{2}{*}{2007} & -41.06 & 2006 & 0.63 \\
\hline & & 2007 & 5.04 \\
\hline
\end{tabular}

A pesar de que se encontró relación significativa para la mayoría de los años entre los valores de las empresas calculados con el modelo FED y los valores de mercado, en el cuadro anterior se observa que no hay consistencia en el coeficiente $\beta_{l .}$ Por lo tanto, no existe evidencia que permita inferir que el modelo FED ayuda a determinar el valor de mercado.

Con la finalidad de conocer la intensidad de la asociación lineal entre los valores calculados con el modelo FED y los valores de mercado de las empresas, en el cuadro 3 se muestran las correlaciones. 


\section{Cuadro 3}

\section{Correlación anual entre FED y valor de mercado}

\begin{tabular}{ccc}
\hline Año & $\begin{array}{c}\text { Correlación entre los valores de } \\
\text { mercado y valores calculados con } \\
\text { el último PIB sectorial conocido }\end{array}$ & $\begin{array}{c}\text { Correlación entre los valores de } \\
\text { mercado y los valores calculados con } \\
\text { la media geométrica del PIB sectorial }\end{array}$ \\
\hline 2001 & 0.60 & $\mathbf{0 . 7 7}$ \\
2002 & 0.22 & -0.34 \\
2003 & 0.83 & 0.81 \\
2004 & 0.72 & 0.71 \\
2005 & 0.72 & $\mathbf{0 . 8 0}$ \\
2006 & 0.57 & $\mathbf{0 . 7 2}$ \\
2007 & -0.45 & 0.36 \\
\hline
\end{tabular}

Como se puede observar en el cuadro 3 , las regresiones entre los valores de las empresas calculados con la media geométrica del PIB sectorial y los valores de mercado arrojan coeficientes de correlación más altos para la mayoría de los años en comparación con las otras regresiones. Lo anterior indica que la media geométrica del PIB sectorial proporciona una mejor estimación del crecimiento de las empresas.

El cuadro 4 muestra el $p$-value y $t$ que se obtuvo en cada regresión:

\section{Cuadro 4}

Valores de $p$-value y estadístico $t$ de las regresiones anuales

\begin{tabular}{|c|c|c|c|c|}
\hline \multirow[t]{2}{*}{ Año } & \multicolumn{2}{|c|}{$\begin{array}{c}\text { Valores de mercado y valores } \\
\text { calculados con el último PIB } \\
\text { sectorial conocido }\end{array}$} & \multicolumn{2}{|c|}{$\begin{array}{l}\text { Valores de mercado y los } \\
\text { valores calculados con la media } \\
\text { geométrica del PIB sectorial }\end{array}$} \\
\hline & $t$ & p-value & $t$ & p-value \\
\hline 2001 & 5.2179 & $0.0000 * * * 11$ & 8.2702 & $0.0000 * * *$ \\
\hline 2002 & 1.5486 & 0.1280 & -2.5079 & $0.0156^{* *}$ \\
\hline 2003 & 10.2098 & $0.0000 * * *$ & 9.4468 & $0.0000 * * *$ \\
\hline 2004 & 7.2889 & $0.0000 * * *$ & 7.0099 & $0.0000 * * *$ \\
\hline 2005 & 7.2232 & $0.0000 * * *$ & 9.3340 & $0.0000 * * *$ \\
\hline 2006 & 4.7886 & $0.0000 * * *$ & 7.1584 & $0.0000 * * *$ \\
\hline 2007 & -3.5344 & $0.0009 * * *$ & 2.6901 & $0.0098 * * *$ \\
\hline
\end{tabular}

$11 * * *$ Si el p-value es menor o igual a .01 existe una relación muy significativa.

** Si el p-value es de 01 a .05 existe una relación poco significativa.

* Si el p-value es igual a .05 existe una relación marginalmente significativa. Un p-value mayor a .05 indica que no existe relación entre las variables. 
Resultados de las regresiones para cada sector

Se elaboraron un total de 14 regresiones clasificadas en dos grupos de siete sectores. Recordemos que definimos dos métodos de estimación del PIB esperado, uno es determinado por el último valor conocido del PIB y otro es el resultado de la media geométrica denominado $P_{I B}$. En los sectores Comercio, Comunicaciones y transportes, Construcción, Industria extractiva, Transformación o manufactura, Empresas diversificadas y Otros Servicios se analizaron 77, 49, 35, 21, 112, 42 y 14 observaciones, respectivamente.

En el cuadro 5 se observan los sectores donde se encontró una relación significativa entre los valores de las empresas calculados con el modelo FED y los valores de mercado:

\section{Cuadro 5 \\ Regresiones sectoriales con relación estadísticamente significativa}

\begin{tabular}{lc|cc}
\hline $\begin{array}{c}\text { Valores calculados con el último PIB sectorial } \\
\text { conocido y valores de mercado de las empresas } \\
\text { Sectores }\end{array}$ & $\begin{array}{c}\text { valores calculados con la media geométrica del PIB } \\
\text { Pectorial y valores de mercado de las empresas } \\
\text { sen relación significativa }\end{array}$ & $\begin{array}{c}\text { Sectores } \\
\text { de la recta }\left(\beta_{l}\right)\end{array}$ & $\begin{array}{c}\text { con relación significativa } \\
\text { de la recta }\left(\beta_{l}\right)\end{array}$ \\
\hline Comercio & 0.48 & Industria manufacturera & 0.79 \\
Comunicaciones & -26.62 & Comercio & 0.66 \\
Construcción & 1.84 & Construcción & 5.50 \\
Minería & 1.10 & Minería & 1.31 \\
\hline
\end{tabular}

A pesar de que se encontró relación significativa entre los valores calculados con el modelo FED y los valores de mercado en la mayoría de los sectores analizados, el coeficiente $\beta_{1}$ no es consistente; esto significa que no existe evidencia que permita inferir que el modelo FED ayuda a determinar el valor de mercado.

La asociación lineal entre los valores de las empresas calculados con el modelo FED y los valores de mercado para cada sector se muestra en el cuadro 6 representada por sus correlaciones: 


\section{Cuadro 6 \\ Correlación sectorial entre FED y valor de mercado}

\begin{tabular}{lcc}
\hline \multicolumn{1}{c}{ Sector } & $\begin{array}{c}\text { Correlación entre los valores } \\
\text { calculados con el último } \\
\text { PIB sectorial conocido y los } \\
\text { valores de mercado }\end{array}$ & $\begin{array}{c}\text { Correlación entre los valores } \\
\text { calculados con la media } \\
\text { geométrica del PIB sectorial y } \\
\text { los valores de mercado }\end{array}$ \\
\hline Manufactura & 0.15 & $\mathbf{0 . 3 9}$ \\
Comercio & -0.35 & 0.29 \\
Construcción & 0.34 & $\mathbf{0 . 4 5}$ \\
Comunicaciones & 0.34 & 0.16 \\
Empresas diversificadas & 0.02 & $\mathbf{0 . 1 6}$ \\
Minería & 0.47 & $\mathbf{0 . 8 3}$ \\
Otros servicios & 0.11 & 0.06 \\
\hline
\end{tabular}

Como se observa en el cuadro 6, las regresiones entre los valores calculados con la media geométrica del PIB sectorial y los valores de mercado de las empresas arrojan coeficientes de correlación más altos para la mayoría de los sectores en comparación con las otras regresiones. Por lo tanto, la media geométrica del PIB sectorial proporciona una mejor estimación del crecimiento de las empresas.

En el siguiente cuadro 7 se muestra el p-value y t que se obtuvieron en cada regresión.

\section{Cuadro 7}

Valores de $p$-value y estadístico $t$ de las regresiones sectoriales

\begin{tabular}{lcc|cc}
\hline Sector & $\begin{array}{c}\text { Valores calculados con el último } \\
\text { PIB sectorial conocido y valores de } \\
\text { mercado }\end{array}$ & $\begin{array}{c}\text { Valores calculados con la media } \\
\text { geométrica del PIB sectorial y valores } \\
\text { de mercado }\end{array}$ \\
\cline { 2 - 5 } & $\boldsymbol{t}$ & $\boldsymbol{p}$-value & $\boldsymbol{t}$ & $\boldsymbol{p}$-value \\
\hline Manufactura & 1.6107 & 0.1101 & 4.3815 & $0.0000^{* * *}$ \\
Comercio & 3.2491 & $0.0017^{* * * 12}$ & 2.6737 & $0.0092^{* * *}$ \\
Construcción & 2.0995 & $0.0434^{* *}$ & 2.9135 & $0.0064^{* * *}$ \\
Comunicación & -2.4967 & $0.0161^{* *}$ & 1.0936 & 0.2797 \\
Empresas diversificadas & 0.1229 & 0.9028 & 1.0154 & 0.3162 \\
Minería & 2.3015 & $0.0328^{* *}$ & 6.0144 & $0.0000^{* * *}$ \\
Otros servicios & 0.3803 & 0.7103 & 0.1987 & $0.8458^{*}$ \\
\hline
\end{tabular}

12*** Si el $p$-value es menor o igual .01 existe una relación muy significativa.

** Si el p-value es de .01 a .05 existe una relación poco significativa.

* Si el p-value es igual a .05 existe una relación marginalmente significativa.

Un p-value mayor a .05 indica que no existe relación entre las variables. 
Los diagramas de dispersión para los sectores donde se encontró una relación muy significativa entre los valores de las empresas calculados con la media geométrica del PIB sectorial y los valores de mercado son los siguientes:
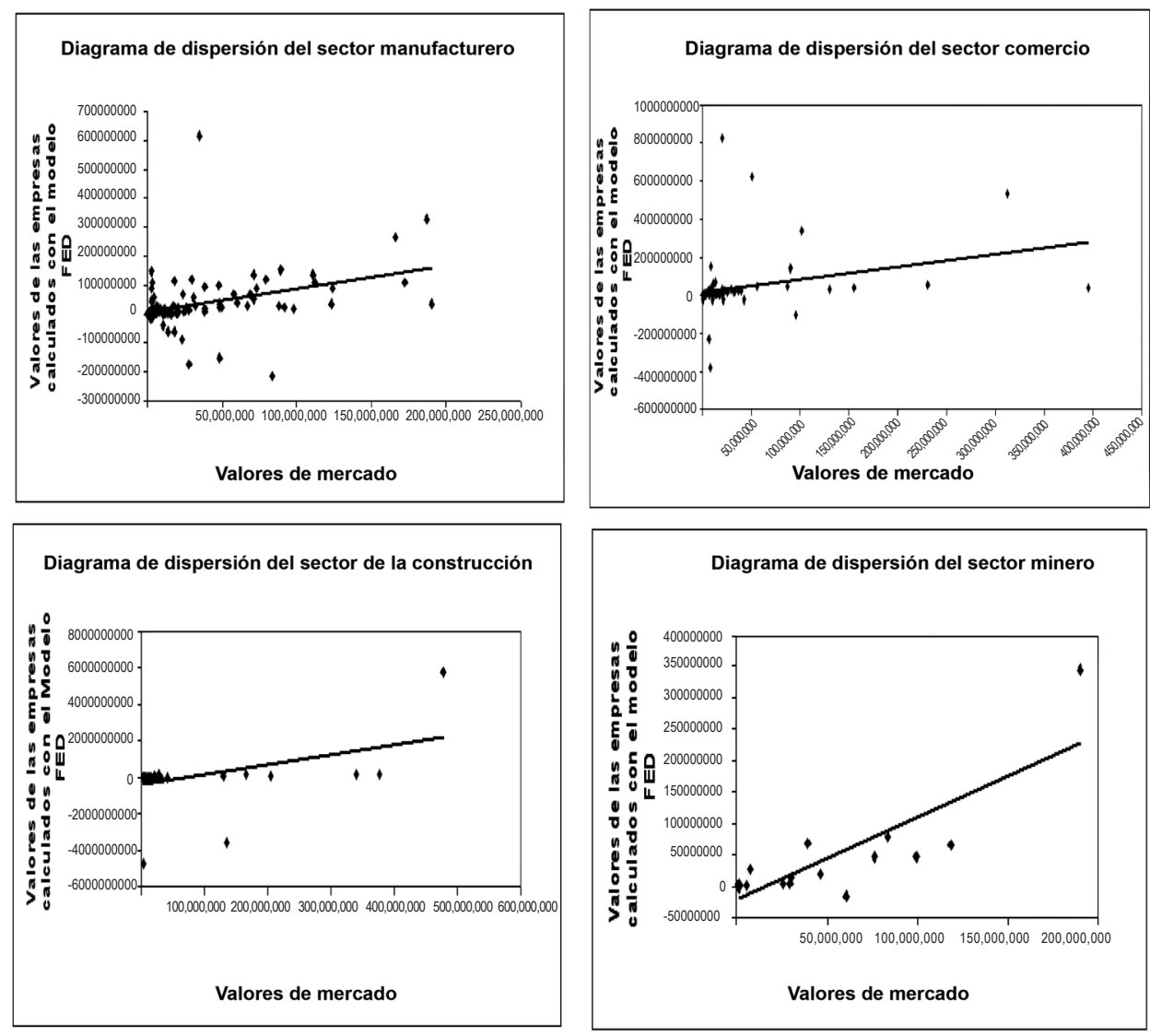

\section{Regresión global}

Se realizaron dos regresiones globales: la primera regresión se hizo entre los valores de las empresas calculados con el último PIB sectorial conocido y los valores de mercado y la segunda entre los valores calculados con la media geométrica del PIB sectorial y los valores de mercado. En las dos regresiones se analizaron 350 observaciones. 
Con un p-value de 0.000 y una $t$ de -4.65 se encontró que existe una relación muy significativa entre los valores de las empresas calculados con el modelo FED utilizando el último PIB sectorial conocido como tasa de crecimiento y los valores de mercado. Esta regresión muestra una correlación de .24.

Por otra parte, con un $p$-value de 0.000 y una $t$ de 4.92 se encontró que existe una relación muy significativa entre los valores de las empresas calculados con el modelo FED utilizando la media geométrica del PIB sectorial como tasa de descuento y los valores de mercado. La correlación que se obtuvo entre ambas variables es de .26 .

\section{Conclusiones}

Los resultados de las regresiones para cada empresa no muestran evidencia suficiente para determinar la existencia de una relación significativa entre los valores calculados con el modelo FED y los valores de mercado. Esto se debe principalmente a que el número de observaciones no es lo suficientemente grande como para suponer que la distribución muestral se inclina a seguir una distribución normal y como consecuencia se invalidan las pruebas.

Los resultados de las regresiones para cada año de análisis entre los valores calculados con el modelo FED utilizando el último PIB sectorial conocido como tasa de crecimiento y los valores de mercado de las empresas indican que hay relación estadísticamente significativa entre ambas variables en los años 2001, 2003, 2004, 2005, 2006 y 2007.

De la misma forma, al correr las regresiones entre los valores de las empresas calculados con el modelo FED, utilizando ahora la media geométrica del PIB sectorial como tasa de crecimiento y los valores de mercado, se encontró que hay una relación estadísticamente significativa entre ambas variables en todos los años que se analizaron. Esto permite inferir que la media geométrica del PIB sectorial proporcionó una mejor estimación de la tasa de crecimiento de las empresas y como consecuencia, mayor asociación lineal entre los valores de mercado y los valores calculados con el modelo FED.

Los resultados de las regresiones para cada sector también son satisfactorios. En los sectores: comercio, comunicación y transporte, construcción y minería se en- 
contró una relación significativa entre los valores calculados con el modelo FED utilizando el último PIB sectorial conocido como tasa de crecimiento y los valores de mercado de las empresas.

También los resultados de las regresiones indican que en los sectores: industria manufacturera, comercio, construcción y minería, tienen una relación estadísticamente significativa entre los valores calculados con el modelo FED utilizando la media geométrica del PIB sectorial como tasa de crecimiento y los valores de mercado de las empresas. Además, se encontró que los coeficientes de correlación de estas regresiones muestran mayor asociación lineal entre ambas variables. Por consiguiente, se deduce, una vez más, que la media geométrica del PIB sectorial proporcionó una mejor estimación del crecimiento de las empresas.

A pesar de que se encontró relación estadísticamente significativa entre los valores calculados con el modelo FED y los valores de mercado de las empresas en la mayoría de los años y sectores analizados, el coeficiente $\beta_{1}$ no fue consistente. Por otra parte, se deduce que la media geométrica del PIB sectorial proporcionó una mejor estimación de la tasa de crecimiento de las empresas y como consecuencia mayor asociación lineal entre los valores de mercado y los valores calculados con el modelo FED.

\section{Referencias}

Adam, J. A. (2005). "Los métodos de valuación de empresas y su relación con la capacidad de las organizaciones para generar valor". Contaduría y Administración, No. 217, septiembre-diciembre.

Anderson, D., Sweeny, D. y Williams, Th. (2004). Estadística para administración y economía. México: Thomson.

Athanassakos, G. (2007). "Valuing internet ventures". Journal of business valuation and economic loss analysis, Western Ontario, Vol. 2, Issue 1.

Banco de México (2008). "Reportes anuales del Banco de México". Recuperados el 26 agosto de 2008. http://www.banxico.gob.mx 
Bolsa Mexicana de Valores (2008). "Reportes anuales de la Bolsa Mexicana de Valores". Recuperados el 30 de agosto de 2008. http://www.bmv.com.mx

Berkman, H. (2000). "The accuracy of Price-Earning and Discounted Cash Flow Methods of IPO Equity Valuation". Journal of International Financial Management and Accounting, New Zeland.

Copeland, T. (2000) Valuation: Measuring and managing the value of companies, $3^{\text {er }}$ ed., EUA: Mc. Kinsey \& Company, Inc., John Wiley \&Sons, Inc.

$\longrightarrow$, Koller, T. and Murrin, J. (1994). Valuation: Measuring and managing the value of companies. EUA: Mc. Kinsey \&Company.

Desmet, D. (2000). "Valuing Dot-Coms”. The Mckinsey Q., No. 1, EUA

Fernández, P. (2004). "How to value a seasonal company discounting cash flows". Social Science Research Network. Working Paper. Madrid:

(2008a). "Métodos de valuación de empresas". Social Science Research Network. Working Paper, No. 1267987, Madrid

(2008b). "Cash flow is a fact. Net income is just an opinion". Social Science Research Network. Working Paper, No. 330540, Madrid

Gitman, L. (2007). Principios de administración financiera. México: Pearson Addison Wesley.

Heer, M. and Koller, T. (2000). "Valuing Cyclical Companies". The McKinsey Quarterly, No. 2, EUA.

James, M. and Koller, T. (2000). "Valuation in emerging markets". The McKinsey Quarterly, No. 4, EUA.

Levine, D. Timothy, K. and Mark, B. (2006). Estadística para administración. México: Pearson Prentice Hall.

López, G. (2000). "El cash flow de la firma y la proyección de los estados financieros". Instituto Argentino de Ejecutivos de Finanzas, No 168, agosto. 
(2001). "Métodos de valuación por descuento de flujos". Instituto Argentino de Ejecutivos de Finanzas, No. 176, diciembre.

(2002). "Valuación de empresas por descuento de flujos: equivalencia y consideraciones prácticas", Instituto Argentino de Ejecutivos de Finanzas, No. 179, junio.

Luehrman, T. (1997). "Using APV: A Better Tool for Valuing Operations". Harvard Businees Review, EUA, mayo-junio.

Mankiw, G. (2004). Principios de economía. España: Mc. Graw Hill.

Nelson, J. (2000). Opposing Discounted Cash Flow Análisis. Defense Counsel Journal, Vol. 67, No. 4, EUA.

Rappaport, A. (1986). Creating shareholder value. EUA: The Free Press.

Saavedra, M. (2007). "La valuación de empresas cotizadas en México, mediante la metodología del modelo de flujo de efectivo disponible". Contaduría y Administración, No. 223, septiembre-diciembre

(2008). Valuación de empresas: metodología para su aplicación. México: Gasca-Sicco.

Sabal, J. (2007). “WACC or APV?". Journal of Business Valuation and Economic Loss Analysis, Vol. 2. Issue 2. España.

Siu, C. (2001). Valuación de empresas: proceso y metodología. México: Instituto Mexicano de Contadores Públicos.

Vélez-Pareja, I. and Tham, J. (2003). "Do the RIM (Residual Income Model), EVA(R) and DCF (Discounted Cash Flow) Really Match?". Social Science Research Network, No. 421100.

Vélez-Pareja, I. (2007). "Constructing Cash Flows”. Social Science Research Network, No. 891968. 
ANEXOS 


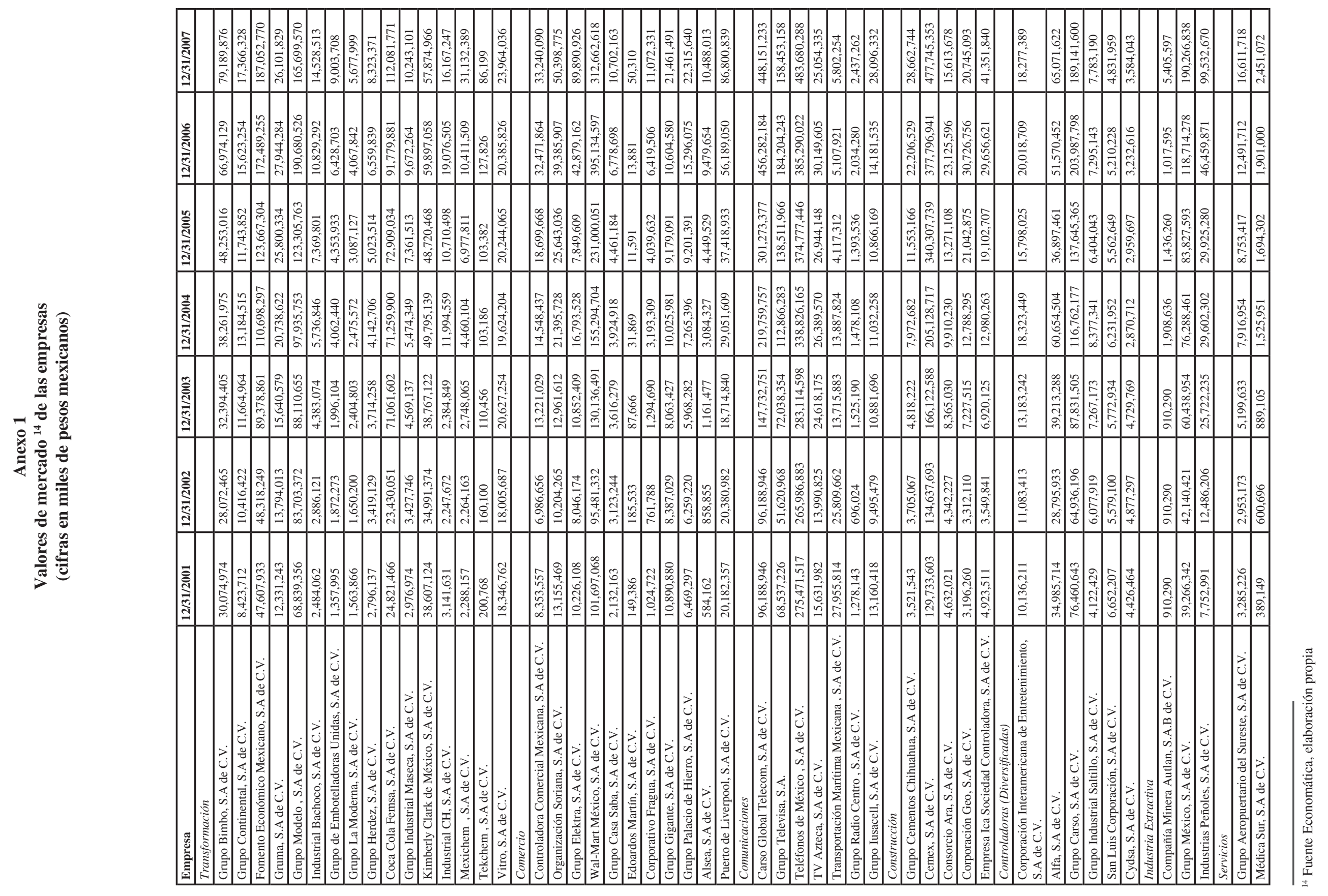




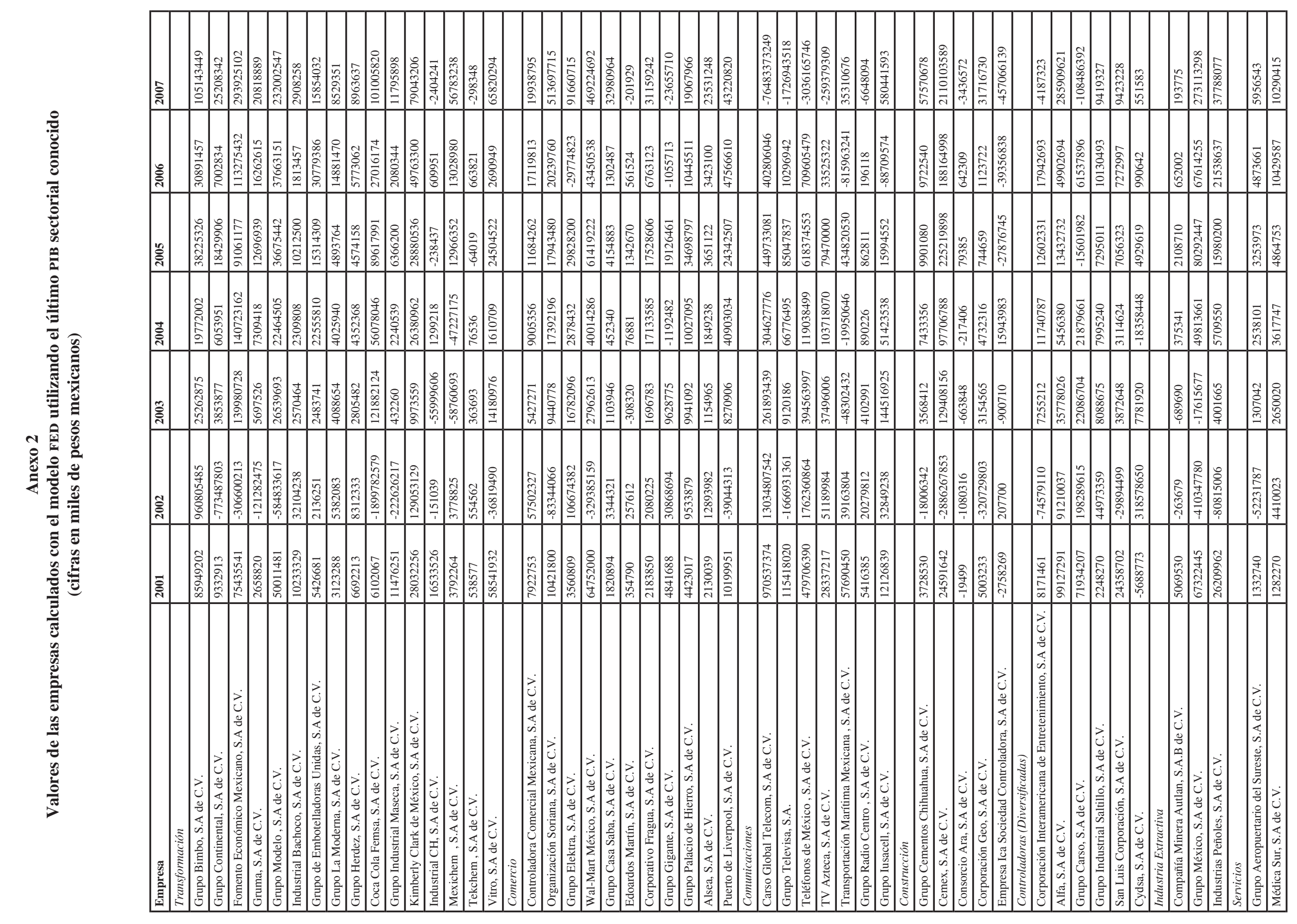




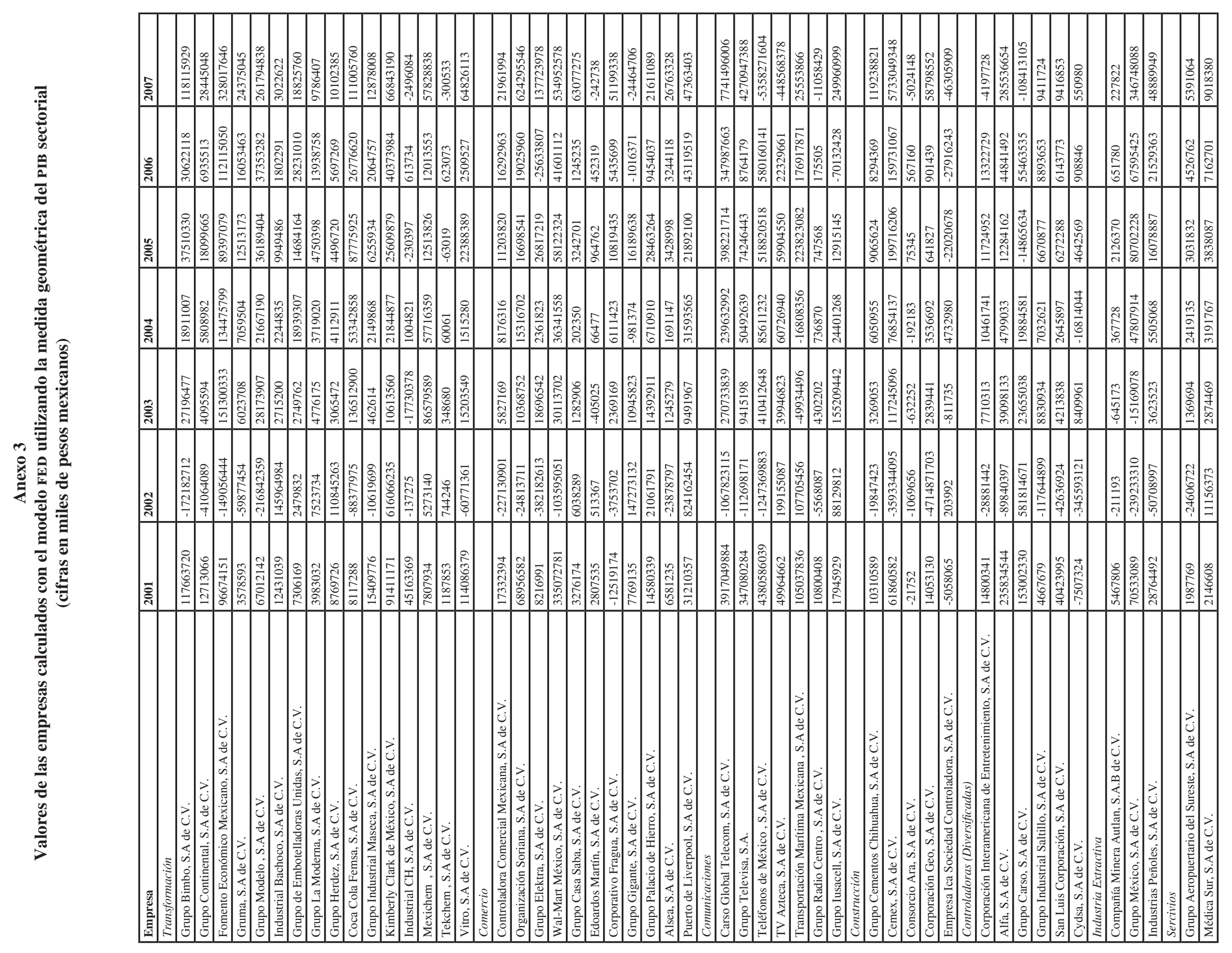

